\section{Atención primaria de salud en Chile: enfermería de práctica avanzada contribuye al acceso universal de salud}

\author{
FRANCISCA AGUIRRE-BOZA ${ }^{1, \mathrm{a}}$, BERNARDITA ACHONDO ${ }^{1, \mathrm{~b}}$
}

\section{Towards universal access to health care: incorporation of advanced practice nurses in primary care}

To move towards universal access to health, the Pan American Health Organization recommends strengthening primary health care (PHC). One of the strategies is to increase the number qualified professionals, both medical and non-medical, working in PHC. In Chile there is a lack of professionals in this level of care, hampering the provision of health. Physicians still prefer secondary and tertiary levels of health. International experience has shown that advanced practice nurses (APN), specialists in PHC are cost-effective professionals able to deliver a complete and quality care to patients. Strong evidence demonstrates the benefits that APN could provide to the population, delivering nursing care that incorporates medical tasks, for example in patients with chronic diseases, allowing greater availability of medical hours for patients requiring more complex management. The success in the implementation of this new role requires the support of the health team, especially PHC physicians, endorsing and promoting the benefits of the APN for the population.

(Rev Med Chile 2016; 144: 1319-1321)

Key words: Advanced Practice Nursing; Health Services Accessibility; Nurse Practitioners; Primary Health Care.
${ }^{1}$ Facultad de Enfermería y

Obstetricia, Universidad de los

Andes, Santiago, Chile.

${ }^{a}$ Enfermera, Magíster en Salud

Pública.

bEnfermera, Magister en Bioética.

Fuente de apoyo financiero:

Fondo de Ayuda a la

Investigación Universidad de los Andes, Chile. La institución no ha tenido influencia sobre la preparación ni revisión del presente documento.

Recibido el 9 de septiembre de 2016, aceptado el 21 octubre de 2016.

Correspondencia a:

Francisca Aguirre Boza

Facultad de Enfermería y

Obstetricia.

San Carlos de Apoquindo 2200,

Las Condes. Santiago, Chile.

faguirre@miuandes.cl

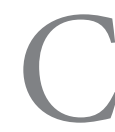

hile necesita avanzar decididamente hacia garantizar el acceso universal en salud. A pesar de las medidas fortalecedoras al sistema, la población aun percibe una crisis en salud, por ejemplo al no tener acceso a una atención de salud integral, oportuna, pertinente, continua y centrada en la persona. La Organización Panamericana de la Salud (OPS) define el acceso universal en salud como "la capacidad de utilizar servicios de salud integrales, adecuados, oportunos y de calidad, en el momento en que se necesitan. (...) se refiere a acciones poblacionales y/o individuales, cultural, étnica, y lingüísticamente apropiadas, con enfoque de género, que tomen en consideración las necesidades diferenciadas para promover la salud, prevenir las enfermedades, prestar atención a la enfermedad (diagnóstico, tratamiento, paliativo y rehabilitación) y ofrecer los cuidados de corto, mediano y largo plazo necesarios" En este sentido, los sistemas de salud basados en la atención primaria en salud (APS) son más eficientes que aquellos basados en especialidades y se reconoce al recurso humano (RRHH) como estratégico para lograr el acceso y la cobertura universal de salud ${ }^{2}$.

El sistema de salud chileno dice estar basado en la APS, pero en la práctica los esfuerzos están principalmente enfocados al nivel hospitalario. La puerta de entrada al sistema debiera ser la APS y cumplir con su rol preventivo y de promoción de la salud, así como mantener a la población sana, o al menos las patologías de base bajo control. Asimismo, disponer de atención médica para resolver de forma oportuna las consultas agudas 
de la población, evitando descompensaciones que requieran atención de urgencia. Sin embargo, la escasez del RRHH que se vive en Chile, especialmente de médicos y profesionales no médicos en el primer nivel de atención, dificulta la provisión de salud. Esto resulta más evidente en zonas rurales y extremas del país.

La OPS en el Reporte "Recursos Humanos para la salud: aumentar el acceso al personal sanitario capacitado en Sistemas de Salud basados en la APS” llamó a los Estados Miembros a implementar estrategias tomadas de experiencias internacionales exitosas, en que se identifican elementos orientadores para alcanzar el acceso universal en salud ${ }^{2}$. Estrategias como aumentar becas para médicos de familia y especialistas, aumentar plazas para el personal médico en APS, mejorar sus condiciones laborales o implementar un sistema de información de RRHH, parecieran no ser suficiente. Los médicos siguen prefiriendo la atención secundaria y terciaria.

En la experiencia internacional, ante escenarios similares de escasez médica en el nivel primario y aumento de la demanda de atención de salud de la población, las enfermeras comienzan a especializarse asumiendo tareas de otras profesiones para atender a la población. Paulatinamente en los años 60, las universidades formalizan estos programas de especialización y surge un nuevo rol de enfermería, que responde a las necesidades de la población en ese contexto ${ }^{3}$. Este nuevo rol es conocido como la enfermera de práctica avanzada (EPA). Inicialmente su ámbito de acción estuvo enfocado principalmente a la APS y en la entrega de una atención integral a los pacientes, familias o comunidades con un mayor nivel de resolutividad $^{3}$. En la actualidad la EPA se ha consolidado y está presente en más de 50 países del mundo y se ha levantado numerosa evidencia que avala la calidad y costo-efectividad en la atención de salud que entrega en comparación con otros profesionales de la salud ${ }^{3}$. Este profesional con formación de magíster, cuenta con un área de especialización, por ejemplo salud familiar, con experiencia y competencias en su ámbito de acción. El Consejo Internacional de Enfermería la define como: "una enfermera especialista que ha adquirido la base de conocimientos de experto, las capacidades de adopción de decisiones complejas y las competencias clínicas necesarias para el ejercicio profesional ampliado, cuyas características vienen dadas por el contexto o el país en el que la enfermera está acreditada para ejercer. Como nivel de entrada se recomienda una maestría" 4 .

Dependiendo del área de especialización o incluso el país donde ejerce, la EPA se reconoce con distintos nombres. La enfermera experta en atención comunitaria/familiar se denomina Nurse Practitioner (NP) $)^{5}$. La EPA tiene dominios generales que le permiten mejorar la provisión de cuidados $^{6}:$ i) La práctica clínica avanzada: otorga atenciones de salud completas desde un modelo holístico de enfermería, con énfasis en la promoción y prevención en salud y asumiendo tareas como el diagnóstico médico, prescripción de cuidados y tratamiento farmacológico y derivación a otros niveles de atención según necesidad; ii) Liderazgo: es capaz de liderar el equipo y acciones de salud desde sus competencias profesionales, su mirada centrada en las necesidades del paciente y cercanía con la comunidad; iii) Perfeccionamiento en la calidad y desarrollo de la práctica clínica: recurre a la mejor evidencia científica disponible para fundamentar la práctica clínica, mejorar procesos y proponer nuevas acciones de cuidado. Asimismo contribuye a la generación de nuevo conocimiento a través de la investigación; iv) Desarrollo personal y de los otros: a través del mentoring interactúa y se encauza al logro de metas elevadas para sí y para el equipo de salud.

Respondiendo al llamado de la OPS, a nivel nacional se organizó un Simposio de EPA para APS (2016), cuyo reporte final describe la contribución de la NP al sistema de salud ${ }^{7}$. Entre ellos, podemos señalar la atención completa a pacientes crónicos derivando al médico exclusivamente pacientes complejos. Esto mejoraría la resolutividad de la APS al ofrecer en una sola prestación cuidados de enfermería y atención médica. De esta forma se optimiza la agenda del médico al disponer de más horas para atenciones de morbilidad u otras prestaciones más complejas, por ejemplo, cirugía menor. En el caso de atención domiciliaria, en una sola prestación la NP podría entregar cuidados de enfermería y además solicitar y/o tomar exámenes, e iniciar tratamiento o modificar el existente oportunamente. Esto le ahorraría al paciente reiteradas visitas a la APS para atención de salud, descongestionando agendas médicas. Para las comunidades que viven en zonas rurales o de difícil acceso, la NP llevaría atención de salud completa y apoyada en telemedicina que podría 
solucionar problemas de salud más complejos. Es así como este profesional mejoraría el acceso a la atención de salud en la APS al mantener a los pacientes crónicos estables evitando episodios agudos y al liberar horas médicas para consulta de morbilidad. De esta manera se podría disminuir las consultas de urgencia a nivel secundario, ya que es sabido que un alto porcentaje de estas atenciones debieran resolverse en el primer nivel de atención. Más aún, en atención de urgencia en APS o en situaciones de emergencia, la NP se visualiza realizando triage, resolviendo problemas de salud de menor complejidad y refiriendo al médico lo más complejo.

En Chile la Asociación Chilena de Educación en Enfermería, junto con asociaciones del gremio y la OPS, están avanzado en implementar el rol de la NP en la APS. Para ello, se están diseñando programas de magíster de EPA con mención comunitaria/ familiar ${ }^{7}$. Se proyecta tener una primera generación de NP para el 2018 y realizar pilotos en distintos lugares de Chile, en un trabajo conjunto con el MINSAL, Municipios y los equipos de salud comunitarios. Asimismo es necesario visualizar el marco legal y ámbito de acción de la EPA, en el contexto de la organización del sistema de salud nacional y las necesidades de la población ${ }^{5}$. La experiencia internacional señala que la práctica debe preceder a la política, es decir primero se debe levantar evidencia para luego normar la práctica. Respecto al ámbito de acción de la NP, se debe definir su rol, responsabilidades y discutir el nivel de complejidad de la atención de salud que otorgue. Una alternativa sería acotarse al manejo de enfermedades agudas comunes y enfermedades crónicas, pacientes postrados, manejo del dolor en crónicos y en cuidados paliativos, y sus respectivas canastas farmacológicas descritas en los planes GES.

Para el éxito de este proyecto, es esencial contar con el apoyo de los médicos, especialmente de APS, que colaboren con la formación de las primeras generaciones, avalen y promuevan los beneficios de la NP para la comunidad. También el apoyo de los tomadores de decisiones de municipalidades y servicios de salud, que a un nivel estratégico permitan la implementación del rol de la NP en beneficio de los pacientes y del sistema de salud como un todo ${ }^{5}$.

\section{Referencias}

1. Organización Panamericana de la Salud. Estrategias para el acceso universal a la salud y la cobertura universal de salud: 53․ Consejo Directivo 66a Sesión del Comité Regional de la OMS para las Américas. Washington, D.C., EUA; OPS, 2014. Reporte No. 14. Disponible en: http:// iris.paho.org/xmlui/handle/123456789/7652 [Consultado el 5 de septiembre de 2016].

2. Organización Panamericana de la Salud. Recursos humanos para la salud: Aumentar el acceso al personal sanitario capacitado en Sistemas de salud basados en

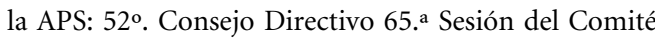
Regional. Washington, D.C., EUA; OPS; 2013. Reporte No. 13. Disponible en: http://iris.paho.org/xmlui/ handle/123456789/4441? show=full [Consultado el 5 de septiembre de 2016].

3. Newhouse RP, Stanik-Hutt J, White KM, Johantgen M, Bass EB, Zangaro G, et al. Advanced Practice Nurse Outcomes 1990-2008: A Systematic Review. Nurs Econ. 2011; 29 (5): 230-51. Disponible en: http://www.ncbi. nlm.nih.gov/pubmed/22372080 [Consultado el 5 de septiembre de 2016].

4. Consejo Internacional de Enfermeras [web]. Ginebra Suiza. CIE; [Consultado el 5 de septiembre 2016]. Definición y características de las funciones de la enfermera de atención directa/enfermería de práctica avanzada. Disponible en: http://www.icn.ch/images/stories/documents/publications/fact_sheets/1b_FS-Enfermera_atencion_directa_practica_avanzada-Sp.pdf

5. Jokiniemi K, Pietila AM, Kylma J, Haatainen K. Advanced nursing roles: A systematic review. Nurs Health Sci. 2012; 14 (3): 421-31. Disponible en: http://onlinelibrary. wiley.com/doi/10.1111/j.1442-2018.2012.00704.x/full [Consultado el 5 de septiembre de 2016].

6. Mantzoukas S, Watkinson S. Review of advanced nursing practice: the international literature and developing the generic features. J Clin Nurs 2007; 16 (1): 28-37. Disponible: http://onlinelibrary.wiley.com/doi/10.1111/ j.1365-2702.2006.01669.x/abstract [Consultado el 5 de septiembre de 2016].

7. Aguirre-Boza F, Cerón C, Soto P. Reporte Simposio La Enfermería de Práctica Avanzada como una Estrategia para alcanzar el Acceso y Cobertura Universal de Salud. Santiago de Chile: Universidad de los Andes-ACHIEEN, 2016. Disponible en: http://media.wix. com/ugd/5598e0_80e35185ffd24ab1ab9bf5d936d1610e. pdf [Consultado el 5 de septiembre de 2016]. 